\section{Tabagismo e fatores associados em adultos: um estudo de base populacional}

\section{Smoking and associated factors in Brazilian adults: a population-based study}

\section{Mirian Kuhnen \\ Antonio Fernando Boing \\ Maria Conceição de Oliveira \\ Giana Zarbato Longo \\ Kathie Njaine}

Programa de Pós-graduação em Saúde Coletiva. Universidade do Planalto Catarinense. Lages, SC.

Pesquisa financiada pelo Programa de Pós-graduação em Saúde Coletiva da UNIPLAC. Agradecimentos: Esse estudo pode ser desenvolvido devido ao esforço e responsabilidade da Dra. Izabella Barison Matos. Agradecemos a contribuição de toda a equipe de trabalho, mestrandos, acadêmicos bolsistas e à Universidade do Planalto Catarinense, que financiou a pesquisa. Correspondência: Mirian Kuhnen. Av. Tancredo Neves, 787, Centro, Correia Pinto, SC CEP: $88535-$ 000.E-mail:kuhnen@uniplac.net

\section{Resumo}

Objetivo: Estimar a prevalência de tabagismo e fatores associados em adultos com idade entre 20 e 59 anos. Métodos: Estudo transversal de base populacional conduzido em uma amostra de 2.022 indivíduos residentes na zona urbana de uma cidade de médio porte do sul do Brasil. Realizaram-se entrevistas domiciliares e foram coletadas informações sobre aspectos socioeconômicos, demográficos, condições auto-referidas de saúde, uso de serviço médico e odontológico, auto-avaliação de saúde, problemas com álcool, estado nutricional e níveis pressóricos. Consideraram-se fumantes os que relataram consumir nos últimos trinta dias qualquer quantidade de fumo. Nas análises bivariadas e multivariada calcularam-se as razões de prevalências (RP) por meio da regressão de Poisson. Resultados: A prevalência global de tabagismo foi de $30,1 \%$ (IC $95 \%: 27,8 ; 32,3)$. As variáveis associadas à maior prevalência de tabagismo na análise multivariada foram: renda mais baixa (RP = 1,49; IC 95\%: 1,26; 1,77), escolaridade menor que oito anos ( $\mathrm{RP}=2,07$; IC 95\%: $1,68 ; 2,56)$, relatar problemas com álcool $(\mathrm{RP}=1,42$; IC 95\%: 1,17; 1,73), não utilizar o serviço médico no último ano $(\mathrm{RP}=1,21$; IC 95\%: 1,$04 ; 1,41$ ) e auto-avaliar a saúde bucal de forma negativa ( $R P=1,28$; IC 95\%: 1,09; 1,50). Condições auto-referidas de saúde associadas ao tabagismo foram: catarro (RP = 2,07; IC 95\%: 1,83; 2,35), chiado no peito $(\mathrm{RP}=1,45$, IC 95\%: 1,20; 1,75), falta de ar $(\mathrm{RP}=1,41$ IC 95\%: 1,19; 1,66) e a eutrofia (RP = 1,57, IC 95\%: 1,36; 1,80). Conclusão: A prevalência de tabagismo na população foi elevada. Políticas públicas são necessárias para diminuir a prevalência e morbidade do tabagismo considerando-se a desigual distribuição dos eventos entre os estratos populacionais.

Palavras-chave: Tabagismo. Estudo epidemiológico. Prevalência. 


\section{Abstract}

Objective: To estimate the prevalence of smoking and associated factors in adults 20 to 59 years old. Methods: A cross-sectional population-based study was carried out in a sample of 2,022 individuals resident in the urban area of a medium-sized city in Southeast Brazil. Home interviews were conducted to collect information about socioeconomic aspects, demographic variables, self-reported health conditions, use of medical and dental services, self-rated health, problems with alcohol, smoking, nutritional status, and blood pressure. Prevalence ratios (PR) through Poisson regression were calculated. Results: The global prevalence of smoking was $30.1 \%$ (CI95\%:27.8-32.3). Variables associated with smoking were: low per capita income (PR = 1.49; 95\% CI:1.26-1.77), less than eight years of education ( $\mathrm{PR}=2.07$; 95\% CI: 1.68-2.56), report of drinking problems ( $\mathrm{PR}=1.42 ; 95 \%$ CI: 1.17-1.73), not having used medical services in the year before $(\mathrm{PR}=1.21 ; 95 \%$ CI: 1.04-1.41), and negative self assessment of oral health ( $\mathrm{PR}=1.28,95 \% \mathrm{CI}$ : 1.09-1.50). Conditions of self-reported health associated with smoking were: sputum ( $\mathrm{PR}=2.07$, 95\% CI: 1.83-2.35), chest wheezing ( $\mathrm{PR}=$ 1.45, 95\% CI: 1.20-1.75), breathlessness (PR $=1.41 ; 95 \%$ CI: 1.19-1.66), and body mass index $<25 \mathrm{~kg} / \mathrm{m}^{2}(\mathrm{PR}=1.57 ; 95 \% \mathrm{CI}$ : 1.36-1.80). Conclusions: The prevalence of smoking in the population was high. Public policies are needed to reduce the prevalence and morbidity of smoking considering the unequal distribution of events in the population strata.

KeyWords: Smoking. Epidemiologic studies. Prevalence.

\section{Introdução}

Durante as últimas décadas o controle do tabagismo configurou-se como um dos maiores desafios da saúde pública. $\mathrm{Na}$ medida em que os fumantes expõem a si mesmos e aqueles que os cercam a inúmeras substâncias tóxicas e que causam doenças respiratórias, cardiocirculatórias e neoplasias, são essenciais políticas públicas que monitorem e desestimulem o consumo de tabaco. Porém, além de constituir hábito social de muitas comunidades, criando-se um contexto de aceitação para seu consumo, o tabagismo é largamente estimulado pela indústria do setor através de agressivas campanhas publicitárias ${ }^{1}$.

Em 2002 havia 1 bilhão de homens e 250 milhões de mulheres fumantes em todo o mundo, sendo a prevalência de tabagismo, em ambos os sexos, bastante superior nos países de média e baixa renda em relação aos países ricos. Além disso, em razão do aumento do consumo de tabaco em determinadas regiões do mundo e como conseqüência do crescimento da população adulta, estima-se que o total de fumantes cresça nos próximos anos e chegue a 2 bilhões em 2030 . O custo econômico do tabagismo nas sociedades também é elevado e deve chegar a US\$ 1 trilhão por ano na década de 2030, valor duas vezes superior ao estimado para $2010^{2}$.

No Brasil, políticas públicas visando reduzir o tabagismo ganharam magnitude a partir de 1989 através do Programa Nacional de Controle do Tabagismo. Daquele ano até 2005 o consumo per capita de cigarros no país foi reduzido em cerca de $32 \%$. Já a prevalência de fumantes na população acima de 18 anos caiu de $34 \%$ em $1989^{3}$ para $22 \%$ em $2003^{4}$ e para $16 \%$ em $2006^{5}$. No entanto, essa variação não tem ocorrido de maneira homogênea entre as regiões e segmentos populacionais do país ${ }^{4-6}$.

Além disso, apesar da importância para a definição de políticas públicas locais e nacionais e para a organização dos serviços de saúde, estudos sobre a prevalência de tabagismo e os fatores associados em cida- 
des de médio porte do Brasil são escassos na literatura. O presente estudo objetivou estimar a prevalência de tabagismo e fatores associados em adultos de um município de médio porte do sul do Brasil.

\section{Método}

Foi conduzido estudo transversal de base populacional em Lages, município situado na serra de Santa Catarina e distante 176,5 km da capital do estado, Florianópolis. Lages é cidade-pólo regional e concentra atividades de referência no comércio, educação e serviços de saúde. Segundo dados do Instituto Brasileiro de Geografia e Estatística (IBGE) ${ }^{7}$, a população estimada do município em 2006 era de 168.382 habitantes. A população alvo do presente estudo foram as pessoas com idade entre 20 e 59 anos de idade (52,0\% da população do município), de ambos os sexos e residentes na área urbana ( $97,4 \%$ da população). O trabalho de campo foi desenvolvido entre os meses de maio e outubro de 2007.

\section{Cálculo e seleção da amostra}

Para o cálculo do tamanho da amostra consideraram-se os seguintes parâmetros: população de referência igual a 86.998 pessoas; nível de confiança de $95 \%$; prevalência esperada de tabagismo de $22 \%$; erro amostral de três pontos percentuais e efeito do desenho igual a dois. Por fim, adicionaram-se $10 \%$ a fim de compensar recusas e perdas e $20 \%$ para o controle de possíveis variáveis de confusão. O cálculo foi realizado através do programa Epi-Info 6.04. O tamanho da amostra necessária foi igual a 1.917 pessoas. Como esta pesquisa está inserida num grande inquérito populacional, onde outros desfechos foram investigados, a amostra final foi ampliada para 2.051 pessoas.

O processo de amostragem foi realizado em dois estágios, sendo utilizado o setor censitário, unidade de recenseamento do IBGE, como primeiro estágio de seleção e o domicílio como segundo. Participaram do estudo 60 setores censitários urbanos (do total de 186), sorteados através de amostragem casual simples, sem reposição. Todos os indivíduos pertencentes à faixa etária do estudo e residentes nos domicílios sorteados foram considerados potenciais participantes da pesquisa.

Indivíduos institucionalizados (prisões, asilos, hospitais e outras) e os inaptos a responder ao questionário por motivo de impedimento físico ou mental foram considerados como critérios de exclusão. Assumiram-se como perdas aqueles que se recusaram a participar da pesquisa e os residentes em domicílios visitados pela equipe de pesquisa no mínimo quatro vezes, incluída ao menos uma visita em finais de semana e outra no período noturno, sem que o entrevistador conseguisse localizar a pessoa sorteada.

Os dados foram coletados por 10 duplas de entrevistadores previamente treinados, e que desconheciam os objetivos do estudo, através de questionário estruturado e pré-testado. O controle de qualidade dos dados foi efetuado pelo telefone através da seleção aleatória de $10 \%$ dos entrevistados. O estudo foi aprovado pelo Comitê de Ética em Pesquisa da Universidade do Planalto Catarinense sob protocolo número 01/2006.

\section{Variáveis do estudo}

A variável dependente deste estudo foi o tabagismo. Aos sujeitos de pesquisa foi indagado se na época da entrevista eram fumantes, ou seja, se nos últimos trinta dias haviam consumido qualquer quantidade de fumo ${ }^{8}$. Aos que respondiam negativamente foi perguntado se alguma vez na vida a pessoa havia fumado regularmente. Assim, as três categorias obtidas foram "nunca fumante", "fumante atual" e "ex-fumante". Para análise do presente estudo, a variável foi analisada de maneira dicotômica, considerando-se os fumantes atuais e os não fumantes atuais (pessoas que nunca fumaram ou que já abandonaram o hábito).

As variáveis socioeconômicas e demográficas analisadas foram sexo, idade (20 a 
29, 30 a 39, 40 a 49 ou 50 a 59 anos), raça/ cor de pele auto-referida (branca ou parda/ preta), renda mensal per capita em salários mínimos categorizada segundo os quartis de sua distribuição $(0,02-0,50,0,51-0,88$, 0,89-1,58 ou 1,59-19,74), escolaridade (em anos de estudo: até 4,5 a 8,9 a 11 e 12 ou mais) e estado conjugal (com ou sem companheiro). Outras variáveis independentes foram uso de serviço médico (consultou o médico no último ano ou não), auto-avaliação da saúde geral e bucal (categorizadas em positiva ou negativa), problema com álcool (mensurado através do instrumento $\mathrm{CAGE}^{9}$ e dicotomizada em sim ou não), níveis pressóricos elevados (pressão arterial maior que $140 \mathrm{mmHg} \times 90 \mathrm{mmHg}^{10}$ e os que referiram uso de medicação anti-hipertensiva no momento da coleta) e estado nutricional (calculado pela razão do peso pela altura ao quadrado; as pessoas foram classificadas segundo critérios da Organização Mundial de Saúde (OMS) ${ }^{11}$ em eutróficos, sobrepesos e obesos). Por fim, foram obtidas informações auto-referidas sobre condições de saúde, perguntando-se aos sujeitos de pesquisa se já haviam apresentado história de diagnóstico médico de derrame cerebral (acidente vascular cerebral-AVC), enfisema, bronquite/ asma, catarro, chiado no peito ou falta de ar nos doze meses anteriores à pesquisa.

\section{Análise estatística}

O banco de dados foi criado no programa Epi Info 6.04 através de dupla digitação e a análise estatística foi realizada no programa Stata 9. Foram realizadas análises bivariadas entre as variáveis dependente e independentes, obtendo-se como medida de efeito as razões de prevalências (RP) por meio da regressão de Poisson ${ }^{12}$. Foram incorporadas na análise multivariada as variáveis com valor de $\mathrm{p}<0,25$ e mantidas no modelo final aquelas com $\mathrm{p}<0,05$.

Como as distribuições etárias e por sexo da amostra investigada apresentavam variações em relação ao descrito pelo IBGE para o município no ano de $2007^{7}$, foram calculados e incorporados pesos de tal modo que a distribuição demográfica da amostra coincidisse com as informações intercensitárias do IBGE. Os cálculos também consideraram o efeito do delineamento amostral. Tais ajustes foram conduzidos utilizando-se o conjunto de comandos svy do Stata.

\section{Resultados}

A taxa de reposta obtida foi igual a $98,6 \%$ ( $n=2.022)$. A média de escolaridade da amostra efetivamente investigada foi de 9,2 anos de estudo (desvio padrão $=4,2$ anos) e a renda familiar média igual a $\mathrm{R} \$ 1.672,40$ (desvio padrão $=\mathrm{R} \$ 1.657,72)$. Verificou-se predomínio do sexo feminino e de pessoas que auto-referiram cor branca (Tabela 1), sendo a média de idade igual a 38 anos (desvio padrão $=11,6$ anos).

A prevalência global de tabagismo foi de 30,1\% [ IC $_{95 \%}$ 27,8-32,3] (Tabela 1). Entre os fumantes, $80 \%$ iniciaram o hábito antes dos 19 anos de idade, com média de 16,6 anos. Observou-se que $58,6 \%$ dos fumantes consumiam, em média, até 18 cigarros por dia, $36,8 \%$ consumiam de 20 a 35 cigarros/ dia e $4,4 \%$ entre 40 e 80 cigarros/dia. $\mathrm{Na}$ população estudada havia, ainda, 15,5\% [IC $\left.{ }_{95 \%} 14,0-16,9\right]$ de ex-fumantes.

$\mathrm{Na}$ análise bivariada (Tabela 2) verificouse que os indivíduos no quartil de menor renda e aqueles com escolaridade menor que oito anos de estudo apresentaram prevalência de tabagismo 2,15 e 2,39 vezes maior que o grupo mais rico e com maior escolaridade, respectivamente. Também as pessoas nas faixas etárias intermediárias, os pardos/pretos, com problema com álcool, que não utilizaram o serviço médico e autoavaliaram sua saúde geral e bucal de forma negativa apresentaram maior prevalência de tabagismo. Na análise multivariada, raça/cor, estado conjugal e faixa etária apresentaram $\mathrm{p}>0,05$, porém a última permaneceu no modelo para ajuste. Nas demais variáveis, houve variações nas RPs, porém mantiveram associação estatisticamente significante com o desfecho.

Na Tabela 3 observa-se maior prevalência do desfecho entre os indivíduos com 
Tabela 1 - Distribuição da amostra e prevalência de tabagismo segundo variáveis demográficas, socioeconômicas e de auto-avaliação de saúde. Lages, 2007.

Table 1 - Sample distribution and prevalence of smoking according to demographic, socio-economic and health selfassessment variables. Lages, 2007.

\begin{tabular}{|c|c|c|c|c|}
\hline \multirow[t]{3}{*}{ Variáveis } & \multicolumn{2}{|c|}{ Distribuição da amostra } & \multirow{2}{*}{\multicolumn{2}{|c|}{$\begin{array}{l}\text { Tabagismo } \\
\text { Prevalência }\end{array}$}} \\
\hline & \multirow[t]{2}{*}{$\mathrm{n}$} & \multirow[t]{2}{*}{$\%^{*}$} & & \\
\hline & & & $\%$ & IC $95 \%$ \\
\hline \multicolumn{5}{|l|}{ Sexo $(n=2.022)$} \\
\hline Masculino & 779 & 47,8 & 31,5 & 28,$7 ; 34,3$ \\
\hline Feminino & 1.243 & 52,2 & 28,7 & 25,$9 ; 31,6$ \\
\hline \multicolumn{5}{|c|}{ Faixa etária em anos $(n=2.018)$} \\
\hline $20-29$ & 623 & 32,2 & 23,7 & 19,$0 ; 28,4$ \\
\hline $30-39$ & 444 & 29,7 & 32,5 & 26,$6 ; 38,3$ \\
\hline $40-49$ & 528 & 22,9 & 35,8 & 31,$1 ; 40,4$ \\
\hline $50-59$ & 423 & 15,2 & 30,1 & 23,$9 ; 36,3$ \\
\hline \multicolumn{5}{|l|}{ Raça/cor $(n=2.017)$} \\
\hline Branca & 1.237 & 63,6 & 27,5 & 24,$6 ; 30,3$ \\
\hline Preta e parda & 720 & 36,4 & 34,2 & 31,$2 ; 37,2$ \\
\hline \multicolumn{5}{|c|}{ Renda em salários mínimos (per capita) $(n=1.984)$} \\
\hline $1,59-19,74$ & 467 & 22,6 & 20,4 & 16,$8 ; 23,9$ \\
\hline $0,89-1,58$ & 515 & 26,4 & 27,1 & 21,$9 ; 32,3$ \\
\hline $0,51-0,88$ & 500 & 25,6 & 28,9 & 24,$4 ; 32,9$ \\
\hline $0,02-0,50$ & 502 & 25,3 & 43,7 & 39,$0 ; 48,3$ \\
\hline \multicolumn{5}{|c|}{ Escolaridade (1.995) } \\
\hline$\geq 12$ & 456 & 23,0 & 15,8 & 12,$9 ; 18,7$ \\
\hline $9-11$ & 611 & 31,8 & 25,2 & 21,$9 ; 28,6$ \\
\hline $5-8$ & 571 & 29,3 & 41,9 & 37,$5 ; 46,3$ \\
\hline$\leq 4$ & 357 & 15,9 & 37,9 & 31,$1 ; 44,7$ \\
\hline \multicolumn{5}{|c|}{ Estado conjugal $(n=2.017)$} \\
\hline Com companheiro & 1.410 & 70,0 & 29,5 & 26,$9 ; 32,1$ \\
\hline Sem companheiro & 607 & 30,0 & 28,7 & 24,$0 ; 33,5$ \\
\hline \multicolumn{5}{|l|}{ CAGE $(n=2.022)$} \\
\hline 0 & 1.790 & 86,7 & 28,2 & 25,$7 ; 30,6$ \\
\hline$\geq 1$ & 232 & 13,3 & 42,5 & 36,$2 ; 48,4$ \\
\hline \multicolumn{5}{|c|}{ Uso de serviço médico $(n=2.021)$} \\
\hline Sim & 1.546 & 74,6 & 27,9 & 25,$0 ; 30 ; 4$ \\
\hline Não & 475 & 25,4 & 36,4 & 33,$1 ; 39,8$ \\
\hline \multicolumn{5}{|c|}{$\begin{array}{l}\text { Auto-avaliação da saúde geral } \\
(n=2.022)\end{array}$} \\
\hline Positivo & 1.472 & 75,5 & 27,8 & 24,$9 ; 30,8$ \\
\hline Negativo & 550 & 24,5 & 37,0 & 31,$8 ; 42,2$ \\
\hline \multicolumn{5}{|c|}{ Auto-avaliação da saúde bucal ( $n=2.008)$} \\
\hline Positivo & 1.142 & 56,8 & 25,2 & 22,$6 ; 27,6$ \\
\hline Negativo & 866 & 43,2 & 36,4 & 32,$6 ; 40,3$ \\
\hline \multicolumn{5}{|c|}{ Fumantes $(n=2.016)$} \\
\hline Sim & 600 & 30,1 & 30,1 & $27,8-32,3$ \\
\hline Não & 1.416 & 69,9 & 69,9 & $67,7-72,2$ \\
\hline
\end{tabular}

*Valores ajustados pelo efeito de delineamento e ponderados por sexo e faixa etária.

* Values adjusted by the outlining effect and weighed by gender and age. 
Tabela 2 - Análises bivariada e multivariada entre tabagismo e variáveis demográficas, socioeconômicas e de autoavaliação de saúde. Lages, 2007.

Table 2 - Bivariate and multivariate analyses between smoking and demographic, socio-economic and health selfassessment variables. Lages, 2007.

\begin{tabular}{|c|c|c|c|c|}
\hline Variáveis & RP Bruta (IC 95\%) & Valor $p$ & RP Ajustada (IC 95\%) & Valor $\mathrm{p}$ \\
\hline Sexo & & 0,112 & & * \\
\hline Feminino & 1,00 & & & \\
\hline Masculino & $1,10(0,98-1,23)$ & & & \\
\hline Faixa etária (anos) & & & & 0,103 \\
\hline $20-29$ & 1,0 & 0,021 & 1,0 & \\
\hline $30-39$ & $1,37(1,05-1,79)$ & & $1,22(0,96-1,57)$ & \\
\hline $40-49$ & $1,51(1,20-1,91)$ & & $1,40(1,12-1,76)$ & \\
\hline $50-59$ & $1,27(0,91-1,77)$ & & $1,23(0,89-1,70)$ & \\
\hline Raça/cor & & 0,017 & & * \\
\hline Branca & 1,0 & & & \\
\hline Preta e parda & $1,25(1,09-1,42)$ & & & \\
\hline Renda & & $<0,001$ & & $<0,001$ \\
\hline $1,59-19,74$ & 1,0 & & 1,0 & \\
\hline $0,89-1,58$ & $1,33(1,03-1,72)$ & & $1,17(0,91-1,50)$ & \\
\hline $0,60-0,88$ & $1,42(1,14-1,77)$ & & $1,10(0,89-1,36)$ & \\
\hline $0,02-0,50$ & $2,15(1,82-2,52)$ & & $1,49(1,26-1,77)$ & \\
\hline Escolaridade (anos) & & $<0,001$ & & $<0,001$ \\
\hline$\geq 12$ & 1,0 & & 1,0 & \\
\hline $9-11$ & $1,59(1,31-1,93)$ & & $1,44(1,19-1,73)$ & \\
\hline $5-8$ & $2,65(2,18-3,22)$ & & $2,07(1,68-2,56)$ & \\
\hline$\leq 4$ & $2,39(1,87-3,06)$ & & $1,81(1,40-2,34)$ & \\
\hline Estado conjugal & & 0,425 & & * \\
\hline Com companheiro & 1,0 & & & \\
\hline Sem companheiro & $1,06(0,92-1,23)$ & & & \\
\hline CAGE & & $<0,001$ & & $<0,001$ \\
\hline 0 & 1,0 & & 1,0 & \\
\hline$\geq 1$ & $1,51(1,26-1,80)$ & & $1,42(1,17-1,73)$ & \\
\hline Uso de serviço médico & & $<0,001$ & & 0,008 \\
\hline Sim & 1,0 & & 1,0 & \\
\hline Não & $1,31(1,15-1,49)$ & & $1,21(1,04-1,41)$ & \\
\hline Auto-avaliação da saúde geral & & & & * \\
\hline Positivo & 1,0 & 0,005 & & \\
\hline Negativo & $1,33(1,10-1,61)$ & & & \\
\hline Auto- avaliação da saúde bucal & & $<0,001$ & & 0,002 \\
\hline Positivo & 1,0 & & 1,0 & \\
\hline Negativo & $1,45(1,26-1,68)$ & & $1,28(1,09-1,50)$ & \\
\hline
\end{tabular}

* Variáveis que não se mantiveram associadas no modelo multivariado.

* Variables that did not remain associated in the multivariate model 
história de diagnóstico médico de enfisema, nos que relataram catarro, chiado no peito, falta de ar e entre os eutróficos. Na Tabela 4 estão apresentados os valores das análises bivariada e multivariada. No modelo ajustado, percebeu-se que a prevalência de tabagismo foi $107 \%$ maior entre os que reportaram catarro, $45 \%$ nos que citaram história de chiado no peito e $41 \%$ naqueles que referiram falta de ar. A prevalência de tabagismo também se manteve mais elevada entre os indivíduos eutróficos em relação aos com sobrepeso ou obesidade.

\section{Discussão}

O critério de seleção da amostra do presente estudo, o uso de instrumentos de coleta de dados validados, o controle de qualidade dos dados e o desconhecimento

Tabela 3 - Distribuição da amostra e prevalência de tabagismo segundo condições auto-referidas de saúde, estado nutricional e níveis pressóricos. Lages, 2007.

Table 3 - Sample distribution and prevalence of smoking according to self-report of health status, nutritional status and blood pressure levels. Lages, 2007.

\begin{tabular}{|c|c|c|c|c|}
\hline \multirow[t]{3}{*}{ Variáveis } & \multicolumn{2}{|c|}{ Distribuição na amostra } & \multirow{2}{*}{\multicolumn{2}{|c|}{$\begin{array}{l}\text { Tabagismo } \\
\text { Prevalência }\end{array}$}} \\
\hline & \multirow[t]{2}{*}{$\mathrm{n}$} & \multirow[t]{2}{*}{$\%^{*}$} & & \\
\hline & & & $\%$ & IC $95 \%$ \\
\hline \multicolumn{5}{|c|}{ Níveis pressóricos elevados $(n=2.019)$} \\
\hline $\operatorname{Sim}$ & 1.459 & 72,1 & 30,0 & 26,$7 ; 33,3$ \\
\hline Não & 560 & 27,9 & 30,1 & 27,$3 ; 32,9$ \\
\hline \multicolumn{5}{|c|}{$\operatorname{AVC}(n=2.018)$} \\
\hline Sim & 30 & 1,2 & 44,4 & $24,1,64,7$ \\
\hline Não & 1.988 & 98,8 & 29,8 & 27,$5 ; 32,1$ \\
\hline \multicolumn{5}{|c|}{ Enfisema $(n=1.993)$} \\
\hline Sim & 52 & 2,3 & 53,9 & 38,$3 ; 69,6$ \\
\hline Não & 1.941 & 97,7 & 29,4 & 27,$2 ; 31,5$ \\
\hline \multicolumn{5}{|c|}{ Bronquite/asma ( $n=2.015)$} \\
\hline Sim & 249 & 12,4 & 30,0 & 27,$8 ; 32,3$ \\
\hline Não & 1.766 & 87,6 & 30,3 & 24,$8 ; 35,7$ \\
\hline \multicolumn{5}{|c|}{ Catarro $(n=2.022)$} \\
\hline Sim & 298 & 14,7 & 62,9 & 58,$6 ; 67,3$ \\
\hline Não & 1.724 & 85,3 & 24,5 & 22,$5 ; 26,5$ \\
\hline \multicolumn{5}{|c|}{ Chiado no peito $(n=2.019)$} \\
\hline Sim & 407 & 20,2 & 50,5 & 45,$1 ; 56,0$ \\
\hline Não & 1.612 & 79,8 & 25,1 & 22,$6 ; 27,6$ \\
\hline \multicolumn{5}{|c|}{ Falta de ar $(n=1.931)$} \\
\hline Sim & 688 & 35,6 & 43,1 & 39,$1 ; 47,2$ \\
\hline Não & 1.243 & 64,4 & 23,8 & 21,$2 ; 26,5$ \\
\hline \multicolumn{5}{|c|}{ Estado nutricional ( $n=1.969$ ) } \\
\hline Obeso & 462 & 24,0 & 23,5 & 20,$2 ; 26,8$ \\
\hline Sobrepeso & 672 & 27,6 & 28,4 & 25,$1 ; 31,6$ \\
\hline Eutrófico & 835 & 34,6 & 33,8 & 30,$4 ; 37,1$ \\
\hline
\end{tabular}

* Valores ajustados pelo efeito de delineamento e ponderados por sexo e faixa etária

* Values adjusted by the outlining effect and weighed by gender and age. 
Tabela 4 - Análises bivariada e multivariada entre tabagismo e condições auto-referidas de saúde, estado nutricional e níveis pressóricos. Lages, 2007.

Table 4 - Bivariate and multivariate analyses between smoking and self-report of health status, nutritional status and blood pressure levels. Lages, 2007.

\begin{tabular}{|c|c|c|c|c|}
\hline Variáveis & RP Bruta (IC 95\%) & $\mathrm{p}$-valor & RP Ajustada (IC 95\%) & $\mathrm{p}$-valor \\
\hline \multicolumn{5}{|c|}{ Níveis pressórica elevados } \\
\hline 0,964 & & * & & \\
\hline Não & 1,0 & & & \\
\hline Sim & $1,00(0,87-1,14)$ & & & \\
\hline AVC & & 0,073 & & * \\
\hline Não & 1,0 & & & \\
\hline Sim & $1,49(0,96-2,30)$ & & & \\
\hline Enfisema & & $<0,001$ & & * \\
\hline Não & 1,0 & & & \\
\hline Sim & $1,84(1,39-2,42)$ & & & \\
\hline Bronquite/asma & & 0,933 & & * \\
\hline Não & 1,0 & & & \\
\hline Sim & $1,01(0,85-1,20)$ & & & \\
\hline Catarro & & $<0,001$ & & $<0,001$ \\
\hline Não & 1,0 & & 1,0 & \\
\hline Sim & $2,57(2,35-2,82)$ & & $2,07(1,83-2,35)$ & \\
\hline Chiado no peito & & $<0,001$ & & $<0,001$ \\
\hline Não & 1,0 & & & \\
\hline Sim & $2,01(1,73-2,34)$ & & $1,45(1,20-1,75)$ & \\
\hline Falta de ar & & $<0,001$ & & $<0,001$ \\
\hline Não & 1,0 & & 1,0 & \\
\hline Sim & $1,81(1,56-2,10)$ & & $1,41(1,19-1,66)$ & \\
\hline IMC & & $<0,001$ & & $<0,001$ \\
\hline Obeso & 1,0 & & 1,0 & \\
\hline Sobrepeso & $1,15(0,97-1,35)$ & & $1,26(1,08-1,47)$ & \\
\hline Eutrófico & $1,44(1,22-1,71)$ & & $1,57(1,36-1,80)$ & \\
\hline
\end{tabular}

* Variáveis que não se mantiveram associadas no modelo multivariado

* Variables that did not remain associated in the multivariate model

dos entrevistadores sobre o objetivo do estudo reforçam a validade interna do mesmo. A sobre-representação feminina e da população com idade mais elevada na amostra pode ser citada como uma limitação do estudo, porém foi corrigida nas análises com a incorporação de pesos para ajuste. Também é importante destacar que a definição de "tabagismo" é bastante diversa na literatura e tal fato limita comparações diretas entre estudos. O delineamento transversal da pesquisa permitiu identificar a prevalência do tabagismo na população adulta e os fatores associados ao mesmo, porém apresenta como limitação o possível viés de causalidade reversa uma vez que desfecho e demais fatores são investigados num mesmo momento ${ }^{13}$.

A prevalência do tabagismo encontrada no presente estudo foi de $30,1 \%$, valor quase duas vezes superior à média nacional (16,2\%) observada pelo inquérito telefônico 
VIGITEL $^{5}$ em 2006. A proporção de fumantes em Lages também foi superior a todas as capitais investigadas pelo Instituto Nacional do Câncer através de inquérito populacional conduzido em 2002-2003. Em relação aos países europeus, os valores entre os homens foram inferiores aos descritos nos países da antiga União Soviética $(43,3 \% \text { a } 65,3 \%)^{14}$ e similares a Israel $(32,0 \%)$, Luxemburgo $(32,0 \%)$ e Holanda $(35,0 \%)^{15}$. Para as mulheres, os valores foram substancialmente mais elevados que os dos países da antiga União Soviética (9,3\% a 15,5\%), Espanha (22,0\%), Suécia $(18,0 \%)$ e França $(21,0)$. Já a prevalência de ex-fumantes em Lages (15,5\%) foi inferior à média nacional observada em $2006(22,1 \%)^{5}$.

Além de estarem em patamares altos, as prevalências de tabagismo entre homens e mulheres não foram estatisticamente diferentes. Não há série histórica que permita descrever a tendência de tabagismo em Lages, mas tal achado pode ser oriundo de um acréscimo da exposição tabagística entre as mulheres, fato observado em anos recentes no Brasil ${ }^{4}$. A inserção das mulheres no mercado de trabalho e a adoção de hábitos de vida anteriormente restritos ou preponderantes entre os homens são fatores usualmente citados para explicar a elevação do tabagismo no sexo feminino. Além disso, são citadas as campanhas publicitárias da indústria do tabaco, que tornaram o cigarro um símbolo de emancipação feminina, em geral envolvendo o uso de modelos atléticos, que associavam beleza e liberdade de expressão ao cigarro ${ }^{4}$.

O gradiente positivo entre idade e tabagismo até o estrato de 49 anos, decrescente no grupo seguinte (50-59 anos), é semelhante aos dados do VIGITEL ${ }^{5}$. Provavelmente as campanhas educativas influenciam os jovens atuais a não fumarem e sujeitos de idade madura são estimulados à cessação do fumo. Numa coorte na Inglaterra, Doll et $\mathrm{al}^{16}$ verificaram que, embora $70 \%$ dos sujeitos da pesquisa fumassem quando tinham entre 25 e 34 anos, só $29 \%$ mantinham o hábito com 50 a 64 anos de idade. Ressaltam-se os benefícios da cessação do tabagismo em diversos períodos da vida, sendo que aqueles que deixaram de fumar com 60, 50, $40 \mathrm{ou}$ 30 anos de idade ganharam 3, 6, 9 e 10 anos de vida, respectivamente ${ }^{16}$.

No presente estudo $80 \%$ dos fumantes e ex-fumantes iniciaram o hábito antes dos 19 anos de idade, proporção superior à descrita no inquérito nacional ${ }^{4}(70 \%)$ e influenciada pela estratégia da indústria do tabaco, que investe expressivas quantias em promoção e propaganda para este grupo etário. Tal iniciação precoce ao tabagismo apresenta sérias conseqüências; afinal, quanto mais precocemente se estabelece o hábito maior o risco de mortes prematuras na meia idade ou na idade madura ${ }^{16}$.

A associação negativa entre tabagismo e escolaridade e renda é concordante com os achados de outros estudos nacionais e internacionais ${ }^{4,5,17-19}$. Políticas públicas têm se mostrado eficazes em reduzir a prevalência do tabagismo; no entanto, paradoxalmente, muitas têm induzido o aumento da desigualdade socioeconômica na exposição ao hábito de fumar ${ }^{20,21}$.Giskes et al. ${ }^{20}$, ao analisarem dados combinados de 98 países europeus ao longo de 15 anos, identificaram que homens e mulheres com menor escolaridade apresentavam maior prevalência de tabagismo, menor tendência de declínio do mesmo e maior quantidade de cigarros fumados. Diferentes hipóteses têm sido descritas para explicar esse fenômeno. Uma o atribui ao menor acesso ao conhecimento sobre os malefícios do cigarro entre os mais desprivilegiados; outra indica a maior dificuldade desse segmento populacional em abandonar o vício por dispor de menor acesso aos serviços de saúde e suporte social; uma terceira considera o cigarro como uma "automedicação" que ajuda a lidar com o estresse e as dificuldades cotidianas, mais presentes entre os mais desprivilegiados, que também dispõem de menos recursos alternativos para lidar com esses desafios $^{21,22}$.

Em relação à cor da pele, Cokkinides et al. ${ }^{23}$ relataram menor chance dos que referiram raça/cor de pele preta já terem sido questionados se eram fumantes, de 
terem sido orientados a parar de fumar e de terem usado alguma ajuda para cessação de tabagismo no último ano. Além disso, tem sido relatada maior prevalência de iniciação do tabagismo entre crianças e adolescentes pretos em comparação aos brancos ${ }^{24}$. As desigualdades socioeconômicas e de raça/cor de pele relatadas no presente estudo requerem novas investigações a fim de subsidiar políticas de saúde que, além de reduzir a prevalência do tabagismo, diminuam as desigualdades na exposição ao cigarro.

Quanto à associação identificada entre tabagismo e problemas com álcool, os resultados desse estudo corroboram os achados de outros pesquisadores ${ }^{6,17}$. No entanto, estes achados devem ser analisados com cautela, pois a exposição pode ser a conseqüência e não a causa, como foi discutido por Chaieb e Castellarin ${ }^{25}$. Desse modo, as campanhas e políticas de saúde devem abordar as duas problemáticas conjuntamente.

A maior prevalência de tabagismo entre os que não consultaram médico no ano anterior à entrevista pode estar relacionada ao fato de entre os não-fumantes estarem incorporados os ex-fumantes nesta pesquisa. Outros estudos descreveram que este grupo apresenta maior freqüência de consultas médicas em relação aos nunca fumantes ${ }^{26}$. Supõe-se que os indivíduos que pararam de fumar consultam mais por estarem mais preocupados com seu estado de saúde ou pararam de fumar por estarem doentes ${ }^{26}$.

A proporção de indivíduos que autoavaliaram sua saúde bucal de forma negativa foi maior nos fumantes $(36,4 \%)$ do que entre os não fumantes $(25,2 \%)$, resultado similar ao descrito por Millar \& Locker $^{27}$ no Canadá. A maior insatisfação com a saúde bucal e também com a saúde geral entre os fumantes pode ser explicada pela alta prevalência de problemas de saúde manifestada em fumantes ${ }^{27-29}$. O tabagismo é importante fator de risco para doenças cardiovasculares $^{30,31}$, respiratórias ${ }^{29}$, neoplasias ${ }^{16,29}$, lesões na mucosa oral ${ }^{28} \mathrm{e}$ doença periodontal ${ }^{28}$.

O tabagismo é geralmente correlacionado positivamente com o risco de desenvol- ver doenças isquêmicas do coração ${ }^{16,30,31}$; no entanto o efeito crônico do mesmo sobre a pressão arterial não está claramente estabelecido. O estudo de Doll et al. ${ }^{30}$, com 50 anos de observação de médicos fumantes, não mostrou relação entre pressão arterial e tabagismo, sendo que este fator não explica o grande número de mortes por doenças vasculares. Em concordância, diversos estudos nacionais ${ }^{32,33}$ não estabeleceram associações significativas entre tabagismo e hipertensão, sugerindo investigações futuras para melhor entendimento desse fator.

Quanto ao AVC, na análise multivariada do presente estudo não se verificou associação com o tabagismo. Esse resultado é discordante da literatura, que tem relatado risco até três vezes maior do agravo entre os fumantes ${ }^{30}$. Sabe-se que o fumo acelera processo de envelhecimento dos vasos arteriais, determinando o aparecimento da aterosclerose precoce, mecanismo básico para a ocorrência desses acidentes ${ }^{29}$. Dessa maneira, pesquisas adicionais podem esclarecer os resultados obtidos. Com relação aos sintomas de catarro, chiado no peito e falta de ar, a associação com o tabagismo pode ser explicada pelos efeitos da fumaça do cigarro no trata respiratório. Os dois principais efeitos da mesma são a inflamação e os efeitos mutagênicos que acarreta. Alguns componentes da fumaça são irritantes ou exercem efeitos tóxicos na via aérea e, assim, podem causar lesão ou morte da célula, inflamação local, diminuição na capacidade de limpeza das vias aéreas, devido aos efeitos tóxicos nos cílios, e hiperplasia das células mucosas, que resulta em aumento da produção de muco. Estas últimas alterações podem levar à retenção de muco, predispor à colonização e infecção das vias aéreas e resultar em exacerbação inflamatória ${ }^{29}$.

Consoante com outro estudo ${ }^{34}$, verificou-se associação entre tabagismo e estado nutricional. Ainda que os mecanismos desta associação não estejam totalmente claros, há evidências de que a nicotina seja o agente primariamente envolvido nesse fenômeno por agir em determinados neu- 
rotransmissores que atuam no controle e na regulação do apetite e da saciedade no hipotálamo ${ }^{35}$. No entanto, a diminuição do peso nos tabagistas não oferece vantagens em termos de saúde.

A prevalência do tabagismo entre a população adulto de Lages foi elevada comparando-se à média nacional. Dentre os muitos determinantes das doenças crônicas não-degenerativas o tabagismo constitui um fator de risco comum e condição modificável mediante mudanças no estilo de vida ou interações terapêuticas. Políticas públicas são necessárias para diminuir a prevalência e morbidade do tabagismo com esforços para a cessação do fumo, sendo necessário considerar a desigual distribuição dos eventos entre os estratos populacionais.

\section{Referências}

1. World Health Organization (WHO). Confronting the Tobacco Epidemic in an Era of Trade Liberalization. WHO/NMH/TFI/01.4; 2001.

2. Mackay J, Eriksen M. The tobacco atlas. Hong Kong: WHO; 2002.

3. Brasil. Ministério da Saúde. Instituto Nacional de Alimentação e Nutrição. Pesquisa nacional sobre saúde e nutrição: perfil de crescimento da população brasileira de o a 25 anos. Brasília; 1990.

4. Brasil. Ministério da Saúde. Instituto Nacional de Câncer. Inquérito domiciliar sobre comportamento de risco e morbidade referida de doenças e agravos não transmissíveis: Brasil, 15 capitais e Distrito federal, 2002-2003. Rio de Janeiro: INCA; 2004. Disponível em: http://www.inca.gov.br/inquerito. [Acessado em $06 \mathrm{de}$ novembro de 2008].

5. Brasil. Ministério da Saúde. VIGITEL Brasil 2006. Vigilância de fatores de risco e proteção para doenças crônicas por inquérito telefônico: estimativas sobre freqüência e distribuição sócio-demográfica de fatores de risco e proteção para doenças crônicas nas capitais dos 26 estados brasileiros e no Distrito Federal em 2006. Brasília: Ministério da Saúde; 2007.

6. Zeilmann E, Nedel F, Sandin G, Costa M. Prevalência e fatores associados ao tabagismo em uma comunidade da Região Sul de Santa Catarina, Brasil. ACM 2005; 34(3): $19-25$.

7. IBGE (Instituto Brasileiro de Geografia e Estatística). Cidades@.Disponível em: http://www.ibge.gov.br/ cidadesat/default.php. [Acessado em 18 de março de 2007]

8. Menezes AMB, Victora CG, Padilla RP. The Platino project: methodology of a multicenter prevalence survey of chronic obstructive pulmonary disease in major Latin American cities. BMC Med Res Methodol [Internet]. 2004 June [cited 2008 dec 20]; 4(15): 1-7.Available from: http://www.biomedcentral.com/1471-2288/4/15. [Acessado em 20 de dezembro de 2008].

9. Masur J, Monteiro MG. Validation of the CAGE alcoholism screening test in a Brazilian psychiatric in patient hospital setting. Braz J Med Biol Res 1983; 16: 215-18.
10. Chobanian AV, Bakris GJ, Black HR, Cushman WC, Lee A Green LA, et al. The seventh report of the Joint National Committee on prevention, detection, evaluation, and treatment of high blood pressure: the JNC 7 report. JAMA 2003; 289(19): 2560-72.

11. World Health Organization (WHO). Obesity: preventing and managing the global epidemic of obesity. Report of the WHO consulation of obesity. Geneva; 1997.

12. Barros AJ, Hirakata VN. Alternatives for logistic regression in cross-sectional studies: an empirical comparison of models that directly estimate the prevalence ratio. BMC Med Res Methodol 2003; 3: 21.

13. Rothman KJ, Greenland S. Modern Epidemiology. Lippincott Williams \& Wilkins: Philadelphia; 1998.

14. Gilmore A, Pomerleau J, McKee M, Rose R, Haerpfer CW, Rotman D, Tumanov S. Prevalence of smoking in 8 countries of the former Soviet Union: results from the living conditions, lifestyles and health study. Am J Public Health 2004; 94(12): 2177-87.

15. World Health Organization (2007). European health for all statistical database. Disponível em: http://www.euro. who.int/hfadb. [Acessado em 02 de setembro de 2008]

16. Doll R, Peto R, Boreham J, Sutherland I. Mortality in relation to smoking: 50 years' observations on male British doctors. BMJ [Internet]. 2004 June [cited 2009 Jan 10]; 328:1519.Available from: http://www.bmj.com/cgi/ reprint/328/7455/1519. [Acessado em 10 de janeiro de 2009].

17. Falcão TJ de O, Costa I do CC. O tabagismo em um município de pequeno porte: um estudo etnográfico como base para geração de um programa de saúde pública. J Bras Pneumol 2008; 34(2): 91-7.

18. Yang T, Li F, Yang X, Wu Z, Feng X, Wang Y et al. Smoking patterns and sociodemographic factors associated with tobacco use among Chinese rural male residents: a descriptive analysis. BMC Public Health [Internet]. 2008 jul [cited 2008 20];8(248);1-7. Available from: http:// www.biomedcentral.com/1471-2458/8/248. [Acessado em 20 de novembro de 2008]. 
19. Bozkurt AI, Şahinöz S, Özçırpıcı B, Özgür S, Şahinöz T, Acemoğlu H, et al. Patterns of active and passive smoking, and associated factors, in the South-east Anatolian Project (SEAP) region in Turkey. BMC Public Health [Internet]. 2006 jan [cited 20 dec 2008];6(15);1-8. Available from: http://www.biomedcentral.com/14712458/6/15/. [Acessado em 20 de dezembro de 2008].

20. Giskes K, Kunst AE, Benach J, Borrell C, Costa G, Dahl E. Trends in smoking behaviour between 1985 and 2000 in nine European countries by education. J Epidemiol Community Health 2005; 59: 395-401.

21. Marmot M, Wilkinson RG. Social Determinants of Health. New York: Oxford Press; 2006.

22. Layte R, Whelan CT. Explaining social class inequalities in smoking: the role of education, self-efficacy, and deprivation. Eur Sociol Rev 2008; DOI 10.1093/esr/ jcn022. [Acessado em 10 de janeiro de 2009].

23. Cokkinides VE, Halpern MT, Barbeau EM, Ward E, Thun MJ. Racial and ethnic disparities in smoking-cessation interventions: analysis of the 2005 National Health Interview Survey. Am J Prev Med 2008; 34(5): 404-12.

24. Ahmed NU, Ahmed NS, Semenya KA, Elzey JD, Larson C, Bennet CR et al. Prevalence and correlates of initiation of smoking behavior among preteen black and white children. J Natl Med Assoc 2004; 96(2): 200-8.

25. Chaieb JA, Castellarin G. Associação tabagismoalcoolismo:introdução às grandes dependências humanas. Rev Saúde Pública 1998; 32(3): 246-54.

26. Capilheira MF, Santos IS. Fatores individuais associados à utilização de consultas médicas por adultos. Rev Saúde Pública 2006; 40(3): 436-43.

27. Millar WJ, Locker D. Smoking and oral health status. JCDA 2007; 73: 155-67.
28. Winn D. Tobacco use and oral disease. J Dental Educ 2001; 65(4): 306-12.

29. Torres, BS; Godoy I de. Doenças tabaco-relacionadas. $J$ Bras Pneumol 2004; 30(S 2): 3-7.

30. Doll R, Peto R, Wheatley K, Gray R. Mortality in relation to smoking: 40 years' observations on British doctors. $B M J$ [Internet]. 1994 Oct [cited 2009 Jan 10],309:901-911. Available from: http://www.bmj.com/cgi/content/full/3 09/6959/901?view=long\&pmid=7755693. [Acessado em 10 de janeiro de 2009].

31. Wolf PA, D'Agostino RB, Kannel WB, Bonita R, Belanger AJ. Cigarette smoking as a risk factor for stroke. The Framingham Study. JAMA 1988; 259(7): 1025-9.

32. Piccini RX, Victora CG. Hipertensão arterial sistêmica em área urbana no sul do Brasil: prevalência e fatores de risco. Rev Saúde Pública [Internet]. 1994 [citado em 2009 jan 10],28(4):261-267. Disponível em: http://www. scielosp.org/pdf/rsp/v28n4/04.pdf. [Acessado em 10 de janeiro de 2009].

33. Jardim, PCBV, Gondim MRP, Monego ET, Moreira HG, Vitorino PVO, Souza WKSB et al. Hipertensão arterial e alguns fatores de risco em uma capital brasileira. Arq Bras Cardiol [Internet]. 2007 Abr [citado 2009 mar 14]; 88(4): 452-457. Disponível em: http://www.scielo.br/ pdf/abc/v88n4/en_15.pdf. [Acessado em 14 de março de 2009].

34. Albanes D, Jones DY, Micozzi MS, Mattson ME. Associations between smoking and body weight in the US population: analysis of NHANES II. Am J Public Health 1987; 77: 439-44.

35. Chatkin R, Chatkin JM. Tabagismo e variação ponderal: a fisiopatologia e genética podem explicar esta associação? J Bras Pneumol 2007; 33(6): 712-9.

Recebido em: 24/03/09 Aprovado em: 01/09/09 